\title{
Case finding for the primary prevention of fragility fractures with FRAX (without BMD) in those over 70 years: Reducing the reliance on $\mathrm{BMD}$ as the primary tool
}

Objective(s): To develop and evaluate age-stratified fracture risk score thresholds without dual X-ray
absorptiometry (DXA) to manage osteoporosis.

Methods: A multi-centered cohort study of persons over 70 years recruited from metropolitan General Practices. Participants had osteoporosis risk assessment, DXA, health questionnaire, and FRAX Hip (HF) and Major Osteoporotic fracture (MOF) risk scores without DXA T-scores. Age-stratified fracture risk score thresholds (low, moderate and high risk of osteoporosis) were compared with measured BMD based guidelines for osteoporosis management.

Results: 130/531 (24.5\%) participants had osteoporosis. Our thresholds achieved a correct clinical decision compared to BMD in $83-84 \%$ of cases; avoided $249(56 \%)$ of unnecessary DXAs; and, missed osteoporosis in $5-6 \%$ of cases. The FRAX HF and MOF were $+30 \%$ and $+7 \%$ more accurate than recommended guidelines in detecting osteoporosis.

Conclusions: For those over 70 years of age, our proposed fracture risk score thresholds can reasonably guide osteoporosis management and reduce unnecessary DXA.

Keywords: osteoporosis $\bullet$ osteoporotic fracture $\cdot$ medical history taking

\section{Introduction}

An estimated 2.2 million (10\%) Australians have osteoporosis with prevalence estimates anticipated to rise by $30-40 \%$ to approximately 3 million people by 2021 [1]. Earlier detection of osteoporosis and primary prevention of fragility fractures is paramount in the context of an ageing population $[2,3]$.

Bone mineral densitometry assessment using dual energy X-ray absorptiometry (DXA) is the current gold standard assessment of osteoporosis [4]. In Australia, all people over 70 years of age are eligible for a Medicare subsidised DXA scan. The Pharmaceutical Benefits Schedule (PBS) then provides subsidised anti-osteoporosis agents to those with DXA confirmed osteoporosis (defined as worst site T-score $<-2.5$ ) for primary fracture prevention [5].

An Australian clinical audit called, Bones Beyond 70, evaluated the current General Practitioner (GP) practice of managing of osteoporosis in older patients; and, confirmed low rates of screening [6]. Barriers to adequate patient screening included a lack of access to a DXA machine, particularly in remote or rural Australian communities, i.e. the tyranny of distance, and the out-of-pocket expense of the DXA scan for those under 70 years of age $[6,7]$. DXA possesses some clinical limitations, firstly, it does not measure the tensile strength or quality of the bony structure [8,9]; and, secondly, DXA is known to be unreliable at certain sites, e.g. lumbar spine $\mathrm{T}$-scores in patients with spondyloarthropathy $[8,10]$.

In light of the barriers and clinical limitations of DXA, several risk assessment tools have been developed to predict the absolute probability of fracture. One widely utilised fracture risk calculator is called the, FRAX tool, which incorporates risk factors such as, age, gender, BMI, prior fracture and secondary causes with or without DXA to estimate 10-year fracture probability of a hip fracture and a major

\author{
Charles A Inderjeeth ${ }^{* 1,2,3}$ \& Warren D \\ Raymond ${ }^{1,2}$ \\ 'Department of Rehabilitation and \\ Aged Care, Sir Charles Gairdner and \\ Osborne Park Health Care Group, North \\ Metropolitan Health Service, Western \\ Australia \\ ${ }^{2} \mathrm{Sch}$ ool of Medicine, The University of \\ Western Australia, Perth, Western Australia \\ 3Department of Rheumatology, Sir Charles \\ Gairdner Hospital, North Metropolitan \\ Health Service, Western Australia \\ *Author for correspondence: \\ charles.inderjeeth@health.wa.gov.au
}


osteoporotic fracture at the hip, spine, humerus or wrist $[11,12]$.

In 2008, Kanis et al. [13] developed an algorithm for cost-effective fracture risk management based on age-stratified FRAX fracture risk scores with DXA for patients up to 85 years of age, as opposed to DXA alone or DXA with one or two clinical risk factors [13]. We wanted to adapt this algorithm and stratify patients into clinically meaningful categories according to low, moderate (requiring further investigation) and high risk of having underlying osteoporosis, according to local age-stratified thresholds of FRAX 10-year hip fracture $(\mathrm{HF})$ or major osteoporotic fracture (MOF) scores without DXA. The rationale for this approach is to improve screening and management of osteoporosis in areas without access to DXA and to guide and reassure DXA imaging requests in an environment with scarce economic resources or fiscal restrictions.

The objectives of this study were to use the FRAX HF and MOF risk scores without DXA thresholds to identify people with osteoporosis compared to DXA measurement of BMD T scores. Using age-stratification of a patient's FRAX HF or MOF risk scores, we aimed to estimate (i) low risk patient thresholds: not requiring preventative treatment or further investigation with DXA imaging, thus avoiding unnecessary DXA assessment and cost, (ii) moderate risk patient threshold: with suspected osteoporosis, and (iii) high risk patient threshold: those with osteoporosis, who could commence preventative treatment without the confirmation of osteoporosis by a DXA.

\section{Methods}

This prospective multi-centered cohort study involved collaboration between a tertiary hospital's Fracture Liaison Service and 3 large outer metropolitan General Practices. The latter had a significant proportion of older participants, including those in residential care. One practice was co-located within a residential care facility. This project was approved by the local human research ethics committee.

Eligible participants who were deemed to be at clinical risk of osteoporosis or aged over 70, underwent osteoporosis risk assessment using dual energy X-ray absorptiometry (DXA) scan and health questionnaire. Data was collected on demographics, height, weight, clinical characteristics, and modifiable risk factors as part of the assessment. A mobile DXA machine service was available to each of the 3 outer metropolitan General Practitioner services between April and November in 2010. All scans were performed by a single technician using a single, limited fan beam, Lunar Prodigy machine. FRAX ${ }^{\circledR}$ scores with and without DXA values were calculated for each patient.

Overall total of 935 were enrolled from these 3 primary health care settings Figure 1. We excluded patients with a prior fragility fracture, those with a history or current use of bonesparing medications, those who had taken glucocorticoids for more than 3 months, and those diagnosed with rheumatoid arthritis $(n=404)$. These exclusions were based on our rationale to assess those over 70 for primary screening and without an established diagnosis of osteoporosis or confounding features [14,15]. The final analysis was conducted on a cohort

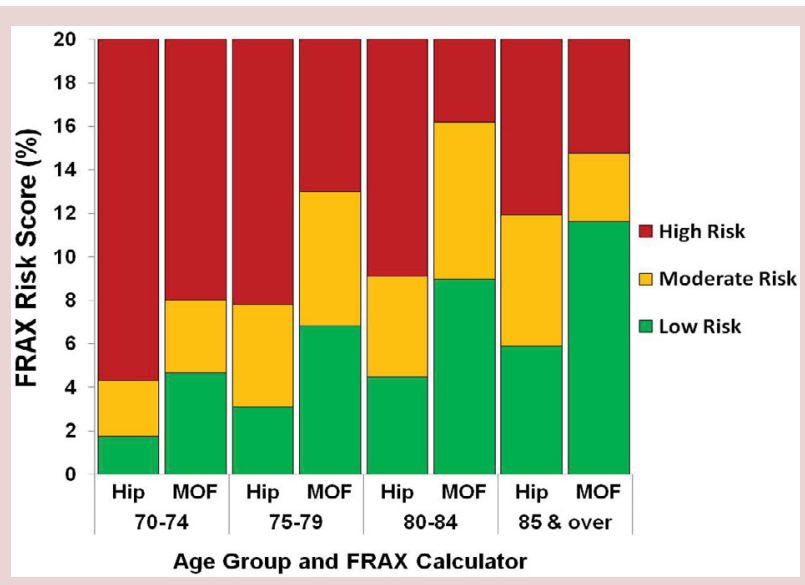

Figure 1. Graphical representation of our proposed age-stratified FRAX Hip and Major Osteoporotic Fracture Risk Score Thresholds. 
naive to fragility fracture and osteoporosis treatment ( $\mathrm{n}=531$ patients), aiming to represent a heterogeneous elderly sample in the primary health care setting without known osteoporosis.

We utilised a modified version of the algorithm proposed by Kanis et al., to calculate clinical thresholds based on age-stratified FRAX fracture risk scores to help detect osteoporosis (defined as DXA measured worst-site T score $<-2.5$ ) without DXA. Subsequently, we determined clinically meaningful thresholds, i.e. low, moderate and high risk of having osteoporosis, anchored to a mean age-stratified FRAX fracture risk score. The aim of these mean age-stratified FRAX fracture risk score thresholds was to capture osteoporosis cases before they experienced a fragility fracture.

A low risk group, i.e. representing the healthiest patients $(\mathrm{n}=302)$ and indicating that this patient would not require further investigation with a DXA or initiated on bone-sparing medication, was set at the mean age-stratified FRAX 10year HF or MOF without DXA, for all patients without clinical risk factors, a BMI $\geq 18.5$ and a DXA T-score $\geq-2.5$. Secondly, an moderate risk group, which represented a FRAX fracture risk score warranting further investigation with DXA, was set at the age-stratified, mean FRAX 10-year fracture risk score without DXA, for persons with no clinical risk factors, a BMI $\geq$ 18.5 and a T-score $<-2.5(\mathrm{n}=91)$. Finally, a high risk group, was set at 1.2 times the moderate risk threshold to minimise the probability that a participant characterised as osteoporotic, based on their FRAX 10-year fracture risk score alone, would be reclassified to the lower threshold with additional information from a DXA scan $(n=138)$. Kanis et al. have demonstrated the effectiveness of age-stratification algorithms to determine clinically meaningful FRAX 10-year fracture score thresholds without DXA, albeit using historical fracture outcomes as opposed to our use of DXA worst site T-score data [13].

A research assistant entered and analysed the data with IBM SPSS V.22. Descriptive statistics included measures of central tendency, frequencies and percentages for the baseline characteristics of the study groups. Receiver Operator Characteristic (ROC) curves and the area under curve (AUC) determined the predictive ability of FRAX - (without DXA) scores in identifying patients with osteoporosis. Osteoporosis was defined as a DXA T-score of $<-2.5$ at the worst site (WS) determined from measures at the femoral neck, total hip, lumbar spine and forearm [11]. Worst site DXA $\mathrm{T}$-score was used in order to capture all patients experiencing low bone density who would have increased risk of a fragility fracture. Given the mixed findings of studies attempting to define the accuracy of DXA to determine osteoporotic $\mathrm{T}$-scores across differing genetic traits, rheumatic disease conditions, environmental stimuli, and hormonal changes with ageing, the use of worst site DXA for the purposes of primary prevention is not unreasonable [16-19].

Finally, the performance of the FRAX 10year HF and MOF risk score thresholds were compared against the National Osteoporosis Foundation's commonly cited 3\% FRAX $\mathrm{HF}$ and 20\% FRAX MOF risk levels for sensitivity, specificity, positive and negative predictive values, and accuracy in the detection of underlying osteoporosis. Furthermore, the proportions of DXA scans saved, the over treatment of non-osteoporotic patients and missed cases of osteoporosis were reported for this older Western Australian cohort.

\section{Results}

A total of 935 participants over 70 years of age were enrolled in the study. Exclusions were applied to participants who had previously sustained a minimal trauma fracture and/or were previously or currently receiving osteoporosis treatment or had other exclusion criteria $(\mathrm{n}=404)$. The final analysis was conducted on 531 patients who were naive to anti-osteoporosis therapy and fragility fractures.

Eligible participants $(\mathrm{n}=531)$ had a mean age of 77.99 years (SD 5.70), with 196 (36.9\%) of patients over 80 years of age, and 238 (44.8\%) females. Risk factors captured at baseline included current (3\%) and previous (12.4\%) tobacco usage, alcohol intake (4.7\%), falls (15.6\%), family history of hip fracture (2.8\%), previous DXA screening (16.6\%), a diagnosis of osteopenia (19.6\%), and DXA confirmed worstsite osteoporosis at baseline (24.5\%) Table 1.

The clinical threshold sub-groups differed for clinical characteristics including, age $(\mathrm{p}<0.001)$, gender $(p<0.001)$, height $(p<0.001)$, weight $(\mathrm{p}<0.001)$ and BMI $(\mathrm{p}<0.001)$. Vitamin D was lowest in the moderate risk group $(\mathrm{p}=0.036)$. Proportion of fallers was not significantly different between groups ( $\mathrm{p}=0.424)$ Table 1 .

There were $130 \quad(24.5 \%)$ osteoporotic participants, defined by worst-site DXA T-score $\leq-2.5$. Receiver Operating Characteristic (ROC) 


\begin{tabular}{|c|c|c|c|c|c|c|}
\hline & & \multirow{3}{*}{$\begin{array}{l}\text { Study Sample } \\
\qquad(n=531)\end{array}$} & \multicolumn{4}{|c|}{ Comparison of the Participants that underpin the thresholds \# } \\
\hline & & & \multirow{2}{*}{$\begin{array}{l}\text { Low Risk } \\
\text { Threshold } \\
\text { (n=302) }\end{array}$} & \multirow{2}{*}{$\begin{array}{c}\text { Moderate Risk } \\
\text { Threshold } \\
(n=91)\end{array}$} & \multirow{2}{*}{$\begin{array}{c}\text { High Risk } \\
\text { Threshold* } \\
(n=57)\end{array}$} & \multirow[t]{2}{*}{ Test } \\
\hline & & & & & & \\
\hline & & MoCT or $n(\%)$ & MoCT or $n(\%)$ & MoCT or $n(\%)$ & MoCT or $n(\%)$ & p-value \\
\hline \multirow{12}{*}{$\begin{array}{c}\text { Clinical } \\
\text { Characteristics }\end{array}$} & Females & $238(44.8 \%)$ & $111(36.8 \%)$ & 66 (72.5\%) & 51 (89.5\%) & \multirow{2}{*}{$<0.001$} \\
\hline & Males & $293(55.2 \%)$ & $191(63.2 \%)$ & $25(27.5 \%)$ & $6(10.5 \%)$ & \\
\hline & Age & $77.99 \pm 5.70$ & $77.42 \pm 5.51$ & $80.35 \pm 5.99$ & $77.58 \pm 5.48$ & $<0.001$ \\
\hline & Height & $166.14 \pm 9.60$ & $168.27 \pm 9.02$ & $160.23 \pm 8.33$ & $158.84 \pm 7.47$ & $<0.001$ \\
\hline & Weight & $75.44 \pm 13.60$ & $78.79 \pm 12.43$ & $66.11 \pm 10.42$ & $66.27 \pm 11.44$ & $<0.001$ \\
\hline & BMI & $27.30 \pm 4.27$ & $27.86 \pm 4.13$ & $25.75 \pm 3.67$ & $26.27 \pm 4.27$ & $<0.001$ \\
\hline & $\begin{array}{l}\text { Currently } \\
\text { smoking }\end{array}$ & $16(3 \%)$ & $0(0 \%)$ & $0(0 \%)$ & $8(14 \%)$ & - \\
\hline & Ex-smoker & 66 (12.4\%) & $19(6.3 \%)$ & $3(3.3 \%)$ & $13(22.8 \%)$ & 0.276 \\
\hline & $\begin{array}{c}\text { Alcohol ( } \geq 3 \\
\text { std. drinks/day) }\end{array}$ & $25(4.7 \%)$ & $0(0 \%)$ & $0(0 \%)$ & $12(21.1 \%)$ & - \\
\hline & $\begin{array}{c}\text { Vitamin D level } \\
(\mathrm{nmol} / \mathrm{L})\end{array}$ & $72.31 \pm 29.80$ & $79.91 \pm 39.75$ & $60.05 \pm 17.90$ & $75.79 \pm 21.43$ & 0.036 \\
\hline & Fallers & $83(15.6 \%)$ & $43(14.2 \%)$ & $14(15.4 \%)$ & $12(21.1 \%)$ & 0.424 \\
\hline & $\begin{array}{l}\text { Falls per } 100 \\
\text { patients }\end{array}$ & 24.8 & 20.9 & 29.7 & 33.3 & - \\
\hline DXA T-score & $\begin{array}{l}\text { Worst-site at } \\
\text { baseline }\end{array}$ & $\begin{array}{l}-1.60(\mathrm{IQR}-2.40 \\
-0.80)\end{array}$ & $\begin{array}{l}-1.20(\text { IQR }-1.90 \\
-0.50)\end{array}$ & $\begin{array}{l}-3.00(\mathrm{IQR}-3.50 \\
-2.70)\end{array}$ & $\begin{array}{c}-2.10(\text { IQR }-3.00 \\
-1.10)\end{array}$ & $<0.001$ \\
\hline \multicolumn{2}{|c|}{$\begin{array}{l}\text { FRAX 10-year hip fracture risk score } \\
\text { with DXA }\end{array}$} & $\begin{array}{l}1.8 \%(\text { IQR } 1.0 \% \\
\text { 3.2\%) }\end{array}$ & $\begin{array}{l}1.4 \% \text { (IQR } 0.8 \% \\
2.3 \%)\end{array}$ & $\begin{array}{l}3.3 \%(\mathrm{IQR} 2.3 \% \\
4.25 \%)\end{array}$ & $\begin{array}{l}2.85 \%(\text { IQR } 1.6 \% \\
5.3 \%)\end{array}$ & $<0.001$ \\
\hline \multicolumn{2}{|c|}{$\begin{array}{l}\text { FRAX 10-year hip fracture risk score } \\
\text { without DXA }\end{array}$} & $\begin{array}{l}3.6 \%(\text { IQR } 2.1 \% \\
5.6 \%)\end{array}$ & $\begin{array}{l}2.9 \%(\mathrm{IQR} 1.8 \% \\
4.0 \%)\end{array}$ & $\begin{array}{c}5.5 \%(\text { IQR } 3.5 \% \\
6.6 \%)\end{array}$ & $\begin{array}{c}8.2 \%(\text { IQR } 5.3 \% \\
14.0 \%)\end{array}$ & $<0.001$ \\
\hline \multicolumn{2}{|c|}{$\begin{array}{c}\text { FRAX 10-year MOF risk score with } \\
\text { DXA }\end{array}$} & $\begin{array}{c}5.4 \%(\text { IQR } 3.8 \% \\
7.7 \%)\end{array}$ & $\begin{array}{l}4.6 \%(\mathrm{IQR} 3.5 \% \\
6.3 \%)\end{array}$ & $\begin{array}{l}8.8 \%(\text { IQR } 6.8 \% \\
11.0 \%)\end{array}$ & $\begin{array}{l}\text { 7.75\% (IQR 5.5\%, } \\
11.0 \%)\end{array}$ & $<0.001$ \\
\hline \multicolumn{2}{|c|}{$\begin{array}{l}\text { FRAX 10-year MOF risk score } \\
\text { without DXA }\end{array}$} & $\begin{array}{c}7.5 \% \text { (IQR 5.2\%, } \\
11.0 \%)\end{array}$ & $\begin{array}{l}6.2 \% \text { (IQR 4.3\% } \\
8.3 \%)\end{array}$ & $\begin{array}{c}11.0 \%(\operatorname{lQR} 7.2 \% \\
14.0 \%)\end{array}$ & $\begin{array}{c}\text { 16.0\% (IQR 11.0\% } \\
23.0 \%)\end{array}$ & $<0.001$ \\
\hline \multicolumn{7}{|c|}{$\begin{array}{l}\text { * High risk threshold includes patients meeting the definition, i.e. participant had FRAX Hip and Major Fracture Risk Scores (\%) that were } \\
\text { both } 1.2 \text { times the moderate risk threshold levels. }\end{array}$} \\
\hline \multicolumn{7}{|c|}{ MoCT: Measures of central tendency } \\
\hline \multicolumn{7}{|c|}{$\begin{array}{l}\text { \# The data presented in Table } 1 \text { for each of clinical threshold groups shows the participants }(n=450) \text { who satisfied the definitions of low } \\
\text { moderate or high risk of osteoporosis as per the Method section. Table } 1 \text { does not show data for all } 531 \text { participants as the heterogeneity } \\
\text { of the cohort meant that they didn't all meet the criteria for the threshold definitions. }\end{array}$} \\
\hline
\end{tabular}

curves and the area under the curve (AUC) determined that FRAX MF and MOF risk scores without DXA exhibited fair predictability (AUC $>70)$ in the detection of worst-site osteoporosis Table 2.

Figure 1 demonstrates the clinical thresholds based on age-stratified, mean FRAX 10-year $\mathrm{HF}$ and MOF risk scores (without BMD) across age groups. The lower threshold signifies that clinicians could safely forgo further investigation or treatment, as osteoporosis is unlikely. The low risk thresholds of FRAX HF and MOF risk scores were $1.75 \%$ and $4.68 \%$ in $70-74$ year olds, $3.11 \%$ and $6.84 \%$ in $75-79$ year olds, $4.50 \%$ and $8.98 \%$ in $80-84$ year olds, and $5.90 \%$ and $11.62 \%$ in individuals 85 years and older. The high risk threshold signifies that clinicians would treat for osteoporosis without the need to undertake additional screening method or a confirmatory DXA scan. The high risk thresholds of FRAX HF and MOF risk scores were $4.31 \%$ and $8.02 \%$ in $70-74$ year olds, $7.81 \%$ and $12.98 \%$ in $75-79$ year olds, $9.09 \%$ and $16.19 \%$ in $80-84$ year olds, and $11.93 \%$ and $14.75 \%$ in individuals 85 years and older. In between the low and high thresholds, i.e. the moderate threshold group signifies that a clinician should order additional screening tests or a confirmatory DXA scan to adequately assess a patient for osteoporosis.

Table 2 shows the clinical decision outcomes of the FRAX 10-year Hip and Major Osteoporotic fracture risk score thresholds for the study sample $(\mathrm{n}=531)$. The thresholds based on the FRAX HF 
risk score without DXA translated into a correct clinical management outcome $84 \%$ of the time, with $11 \%$ of patients potentially "over-treated" and 5\% having their osteoporosis missed ("underestimated"). The thresholds based on the FRAX MOF risk score without DXA resulted in a correct clinical management decision $83 \%$ of the time, with $11 \%$ of patients being "over-treated" and $6 \%$ having their osteoporosis missed.

The characteristics of participants in which the proposed thresholds would miss osteoporosis (5-6\%, Table 3), would be described as having a significant male preponderance $(>90 \%$, $\mathrm{p}<0.001)$, over 80 years of age $(\mathrm{p}<0.001)$, and being osteopaenic or osteoporotic at all measurable sites (median worst site $\mathrm{T}$-score -3.30 (IQR -3.70, -2.90), p<0.001). However, these patients were no more prone to falls or fractures than the other patients groups.

Compared to "universal" cut-offs proposed in the NOF guidelines in the prediction of when to commence treatment of osteoporosis, our thresholds for the FRAX HF risk scores would have lower sensitivity (-20\%) and negative predictive value (NPV) (-4\%), but achieve gains in specificity $(+49 \%)$, positive predictive value (PPV) (+48\%) and accuracy (+30\%). Furthermore, our thresholds for FRAX MOF risk scores would we gain sensitivity $(+11 \%)$, specificity $(+15 \%)$, PPV $(+69 \%)$ and accuracy $(+7 \%)$, with a decrease in NPV $(-8 \%)$ Table 3.

\section{Discussion}

We demonstrate the potential to utilise FRAX without DXA in screening for osteoporosis in a high risk population over 70 years of age. It may play a significant role in screening populations without access to DXA, potentially avoiding unnecessary DXA, and easing costs.

This risk assessment model has a high and acceptable level of accuracy compared to those that incorporate DXA. Assuming the preference for FRAX HF risk score without DXA thresholds are used there is a potential for:

- Making the correct clinical management decision regarding underlying osteoporosis: $83-84 \%$ of the time.

- Avoiding unnecessary DXAs in 249/531 $(55.8 \%)$.

\begin{tabular}{|c|c|c|c|c|}
\hline & & \multicolumn{3}{|c|}{ Clinical Decision } \\
\hline & & Correct & Over treated & OP missed \\
\hline \multirow{2}{*}{$70-74$} & FRAX Hip & $154(84.6 \%)$ & 25 (13.7\%) & $3(1.6 \%)$ \\
\hline & FRAX MOF & $154(84.6 \%)$ & $24(13.2 \%)$ & $4(2.2 \%)$ \\
\hline \multirow{2}{*}{$75-79$} & FRAX Hip & 138 (90.2\%) & $11(7.2 \%)$ & $4(2.6 \%)$ \\
\hline & FRAX MOF & 136 (88.9\%) & $12(7.8 \%)$ & $5(3.3 \%)$ \\
\hline \multirow{2}{*}{$80-84$} & FRAX Hip & 108 (87.8\%) & $10(8.1 \%)$ & $5(4.1 \%)$ \\
\hline & FRAX MOF & $108(87.8 \%)$ & $9(7.3 \%)$ & $6(4.9 \%)$ \\
\hline \multirow{2}{*}{85 \& over } & FRAX Hip & $46(63 \%)$ & $14(19.2 \%)$ & $13(17.8 \%)$ \\
\hline & FRAX MOF & 45 (61.6\%) & $13(17.8 \%)$ & 15 (20.5\%) \\
\hline \multirow{2}{*}{ Total } & FRAX Hip & 446 (84\%) & $60(11 \%)$ & $25(5 \%)$ \\
\hline & FRAX MOF & 443 (83\%) & 58 (11\%) & $30(6 \%)$ \\
\hline
\end{tabular}

Table 3. (a) Accuracy of FRAX in identifying osteoporosis on DXA assessed by Receiver Operating Characteristic (ROC) curves and the area under the curve (AUC); and, (b) comparing our age-specific thresholds to recommended universal NOF cut offs.

\begin{tabular}{|c|c|c|c|c|c|c|}
\hline & a. Raw Scores & \multicolumn{3}{c|}{ b. Our proposed thresholds } \\
\cline { 2 - 7 } & Area Under the Curve & Sensitivity & Specificity & PPV & NPV & Accuracy \\
\hline FRAX HF with DXA & $0.82(95 \% \mathrm{Cl} 0.79,0.85)$ & 1 & 0.28 & 0.31 & 1 & 0.46 \\
\hline FRAX Hip without DXA & $0.74(95 \% \mathrm{Cl} 0.71,0.78)$ & 0.64 & 0.93 & 0.81 & 0.85 & 0.84 \\
\hline FRAX Hip (NOF) 3\% Cut-off & - & 0.84 & 0.44 & 0.33 & 0.89 & 0.54 \\
\hline FRAX Major with DXA & $0.84(95 \% \mathrm{Cl} 0.81,0.87)$ & 0.97 & 0.19 & 0.28 & 0.95 & 0.38 \\
\hline FRAX Major without DXA & $0.75(95 \% \mathrm{Cl} 0.72,0.79)$ & 0.63 & 0.92 & 0.77 & 0.86 & 0.83 \\
\hline FRAX Major (NOF) 20\% Cut-off & - & 0.52 & 0.77 & 0.08 & 0.98 & 0.76 \\
\hline
\end{tabular}


- Potential to miss osteoporosis by not doing a DXA of 5-6\%.

- This approach improved the accuracy of the FRAX HF risk calculator tool $(+30 \%)$, and the FRAX MOF risk calculator tool $(+7 \%)$ when attempting to detect underlying osteoporosis as a primary prevention strategy against fragility fractures.

Currently, the gold-standard assessment for osteoporosis diagnosis and management is the DXA scan in the absence of a fragility fracture. The lack of access to and cost of DXA facilities is a matter of concern for most countries, especially in rural and remote areas. In light of the limited access to screening facilities, better patient selection for DXA and use of alternate screening tools may be a useful adjunct. The WHO has approved the use of tools like FRAX to estimate the fracture risk in different populations based on several clinical risk factors. Although these tools have been validated for different populations across several countries, their reliance on DXA measurement limits their clinical utility, without their own clinically meaningful thresholds, especially in areas where access to such facilities is limited.

We have broadened the clinical utility of FRAX without DXA by proposing age-stratified fracture risk score thresholds that could help clinicians, faced with limited access to DXA facilities, to screen and manage osteoporosis in real-time. The algorithm proposed herein supports the physicians' management of asymptomatic patients, has the potential to reduce unnecessary DXA tests and better targeting those most in need of a DXA. Furthermore, these proposed fracture risk thresholds can be modified as per the methods described to cater to the mix of clinical, economic and social characteristics in a given population.

Although it was not within the scope of this paper there is potential for our model to be cost effective by reducing unnecessary DXA assessments based on an individual's future fracture risk score. This proposition would need to be designed in such a way as to reduce the costs associated with screening and diagnosis whilst simultaneously minimising the number of patients with osteoporosis who are missed (false negatives). A strategy for assessment of osteoporosis based on economic efficiency would ease pressures on scarce health care resources which could be re-directed towards other high- risk groups and reduce the demand for scarce health resources [20].

With evidence of treatment effectiveness well established in populations with both osteoporosis and osteopenia according to fracture outcomes [13], it may be argued that our adaptation of their model, may be more useful in identifying osteoporosis that could be treated without requiring DXA, reducing the susceptibility to fragility fracture(s). This is not withstanding the fact that there is limited evidence for intervention based on risk factors only as we are identifying those with a high likelihood of osteoporotic DXA [21]. The remaining patients will be recommended for DXA screening to identify those with osteoporosis for treatment. The down side of setting a threshold to treat without DXA may be the "overtreatment" of those with osteopenia, without prior fracture. However it could be equally argued that the major fracture burden is in those with osteopenia and those with osteopenia and high FRAX are frailer and at higher risk of fracture, therefore they may benefit from primary prevention. However, this group has limited study data or benefit demonstrated. Apart from costs, the risk to this population of treatment remains low and such a model would ensure that this high risk population is not missed and treatment is started to reduce the risk of fractures and improve bone strength.

\section{Limitations}

One of the potential limitations of our study is the reliance on identifying people with low DXA and lack of prospective follow up of actual fracture occurrence. The literature has demonstrated that low DXA correlates with increased fracture risk over time, but not exclusively, e.g. patients with normal and osteopaenic bone mass also experience increased fracture risk with increasing FRAX scores [22-24]. In addition, FRAX with DXA can accurately identify those who would benefit from treatment for the primary prevention of MTF [22,23]. Another study suggested that FRAX without DXA could be used to pre-screen for osteoporosis to determine who would benefit from DXA [24]. Therefore, attempting to identify those with low DXA is not a critical limitation, as a patient with low DXA and high fracture risk would still require either allied health intervention or commence bone-sparing agents to prevent MTF.

\section{Conclusion}

Osteoporosis is a major concern for several countries with an ageing population. Several 
guidelines and tools (e.g. FRAX) have been developed to guide clinicians on assessment of absolute risk and guide intervention thresholds in equivocal situations. However, reliance of these guidelines and tools on DXA measurement is a limitation especially in areas where such facilities are not readily available and for frailer populations unable to access DXA. Our model aims to reduce this by proposing a model which is based on a fracture risk calculation using FRAX calculator without DXA, and target strategies towards at-risk populations.

Estimating age specific fracture intervention thresholds as we propose has the potential to reduce the number and cost of unnecessary DXAs and reduce the burden on resources that may be scarce or remote in some communities. It may be useful in identifying people who may not require further investigation, who to investigate with BMD and who could reliably be treated without requiring DXA especially where this is not readily available.

\section{Acknowledgements}

The authors wish to thank the following for assistance with access to the database of patients, collection of patient data and supply of DXA reports: Dr. Jennifer Roger, Dr. Therese Fisher and Dr. Robert Will.

\section{Disclosure/Conflict of Interest}

We have not received any funding related to the completion of this research. Dr. Inderjeeth has received honoraria or grants for osteoporosis related work separate from this project from Amgen, Eli Lilly, Merck Sharpe and Dohme, Novartis and Sanofi Aventis in the past.

\section{References}

1. The Burden of Brittle Bones: Epidemiology, Costs \& Burden of Osteoporosis in Australia - 2007. International Osteoporosis Foundation, Osteoporosis Australia (2007).

2. O'Neill S, MacLennan A, Bass S et al. Guidelines for the management of postmenopausal osteoporosis for GPs. Aust. Fam. Physician. 33(11), 910-9 (2004).

3. Port L, Center J, Briffa NK et al. Osteoporotic fracture: missed opportunity for intervention. Osteoporos. Int. 14(9), 780-4 (2003).

4. Cooper C. Invest in your bones, Beat the Break, Know and Reduce your Osteoporosis Risk Factors. International Osteoporosis Foundation 2007.

5. http://www.nps.org.au/publications/healthprofessional/nps-news/2007/maintaining-bone-healthto-prevent-osteoporotic-fractures.
6. Inderjeeth C, Smeath A. Bones beyond 70- Insights into osteoporosis management in the elderly. Aust. Fam. Physician. 39(10), 767-72 (2010).

7. Vondracek SF, Minne P, McDermott MT. Clinical challenges in the management of osteoporosis. Clin. Interv. Aging. 3(2), 315 (2008).

8. Sanders KM, Nicholson GC, Watts JJ et al. Half the burden of fragility fractures in the community occur in women without osteoporosis. When is fracture prevention cost-effective? Bone. 38(5), 694-700 (2006).

9. Lim LS, Hoeksema LJ, Sherin K. Screening for Osteoporosis in the Adult U.S. Population: ACPM Position Statement on Preventive Practice. Am. J Prev. Med. 36(4), 366-75 (2009).

10. Schuit SC, van der Klift M, Weel AE et al. Fracture incidence and association with bone mineral density in elderly men and women: the Rotterdam Study. Bone. 34(1), 195-202 (2004).

11. Kanis JA, Hans D, Cooper C et al. Interpretation and use of FRAX in clinical practice. Osteoporos. Int. 22(9), 2395-411 (2011).

12. Bergh JW, Geel TCM, Lems W et al. Assessment of Individual Fracture Risk: FRAX and Beyond. Curr. Osteoporos. Rep. 8(3), 131-7 (2010).

13. Kanis JA, McCloskey EV, Johansson $\mathrm{H}$ et al. Case finding for the management of osteoporosis with FRAX-assessment and intervention thresholds for the UK. Osteoporos. Int. 19(10), 1395-408 (2008).

14. National Institute for Health and Clinical Excellence. Osteoporosis - Secondary Prevention - Alendronate, etidronate, risendronate, raloxifene, strontium ranelate and teriparatide. United Kingdom: National Institute for Health and Clinical Excellence (2008).

15. Fraser LA, Adachi JD. Glucocorticoid-Induced Osteoporosis: Treatment Update and Review. Ther. Adv. Musculoskelet. Dis. 1(2), 71-85 (2009).

16. Deng X, Wang W, Wu X et al. Correlation between bone mineral density and sexual hormones in healthy Chinese women. J. Environ. Pathol. Toxicol. Oncol. 19(1-2), 167-9 (2000).

17. Namwongprom S, Ekmahachai M, Vilasdechanon N et al. Bone mineral density: correlation between the lumbar spine, proximal femur and Radius in northern Thai women. J. Med. Assoc. Thai. 94(6), 725-31 (2011).

18. Yang TL, Zhao LJ, Liu YJ et al. Genetic and environmental correlations of bone mineral density at different skeletal sites in females and males. Calcif. Tissue. Int. 78(4), 212-7 (2006).

19. Collins GS, Mallett S, Altman DG. Predicting risk of osteoporotic and hip fracture in the United Kingdom: prospective independent and external validation of QFractureScores. BMJ. 342, d3651 (2011).

20. Nshimyumukiza L, Durand A, Gagnon $\mathrm{M}$ et al. An economic evaluation: Simulation of the costeffectiveness and cost-utility of universal prevention strategies against osteoporosis-related fractures. J. Bone. Miner. Res. 28(2), 383-94 (2013). 
21. McClung MR, Geusens P, Miller PD et al. Effect of Risedronate on the Risk of Hip Fracture in Elderly Women. N. Engl. J. Med. 344(5), 333-40 (2001).

22. Hillier TA, Cauley JA, Rizzo JH et al. The WHO Absolute Fracture Risk Models (FRAX): Do Clinical Risk Factors Improve Fracture Prediction in Older Women Without Osteoporosis? J. Bone. Miner. Res. 26(8), 1774-82 (2011).
23. Leslie WD, Lix LM, Johansson $\mathrm{H}$ et al. Independent clinical validation of a Canadian FRAX tool: fracture prediction and model calibration. J. Bone. Miner. Res. 25(11), 2350-8 (2010).

24. Cummins NM, Poku EK, Towler MR et al. Clinical Risk Factors for Osteoporosis in Ireland and the UK A Comparison of FRAX and QFractureScores. Calcif. Tissue. Int. 89(2), 172-7 (2011). 